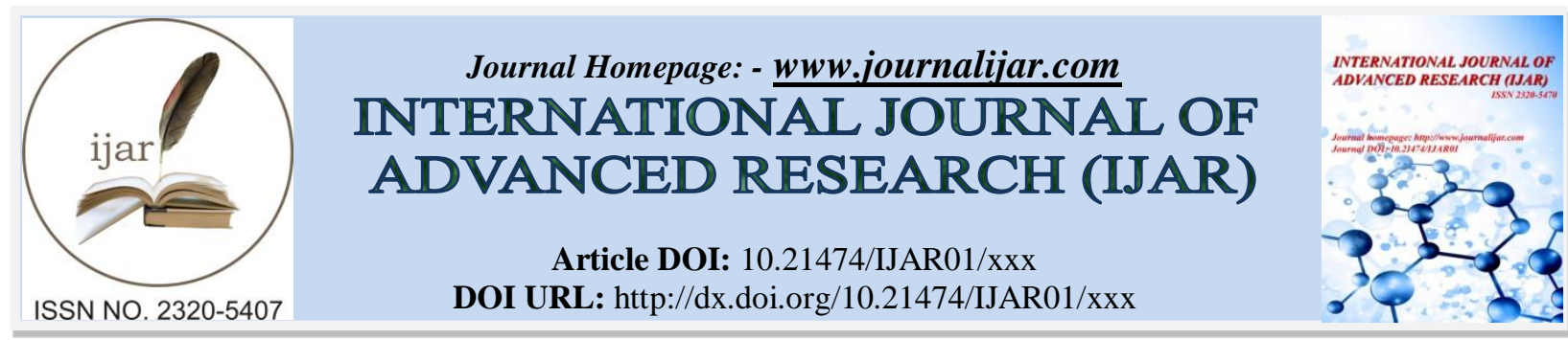

RESEARCH ARTICLE

\title{
PRÉVALENCE, CONNAISSANCES ATTITUDES ET RELATIVES A L'OBÉSITÉ DES FEMMES ENCEINTES PENDANT LES VISITES PRÉNATALES AU CENTRE DE SANTÉ MARIE IMMACULÉE D'ÉTOUG-ÉBÉ (YAOUNDÉ-CAMEROUN).
}

\section{Mbassi Eyebe Josepha Laure, ${ }^{1}$ DAmaris Enyegue Mandob ${ }^{1}$ and Noa Ndoua Claude Cyrille ${ }^{2,3}$.}

1. Département des Sciences Biologiques, Ecole Normale Supérieure de l'Université de Yaoundé I, Yaoundé, Cameroun, BP 047.

2. Faculté de Médecine et des Sciences Biomédicales de l'Université de Yaoundé I, Yaoundé, Cameroun, BP 1364.

3. Centre Hospitalier de Recherche et d'Application de la Chirurgie Endoscopique et de la Reproduction Humaine, Cameroun BP : 5154.

\section{Manuscript Info}

.........................

Manuscript History

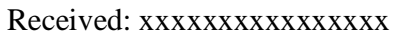
Final Accepted: $x x x x x x x x x x x x$ Published: $\operatorname{xxxxxxxxxxxxxx}$

Key words:-

Obésité, Grossesse, Prévalence,

Connaissances, Attitudes, Pratiques.

\section{Abstract}

Contexte: L'obésité est un problème de santé publique qui est en augmentation fréquente dans le secteur obstétrical. Chez les femmes enceintes, un gain de poids excessif est généralement associé à de nombreuses complications à court et à long terme aussi bien chez la mère que chez l'enfant pendant la grossesse, durant l'accouchement, et après l'accouchement. Au Cameroun en général et à Yaoundé en particulier, il n'existe pas de cadre d'éducation des femmes enceintes relatif à l'obésité et il n'existe pas des études publiées établissant le niveau de connaissance des femmes enceintes vis-à-vis de cette maladie. L'objectif de notre étude est d'évaluer le niveau de connaissance sur l'obésité des femmes enceintes.

Matériel et Méthodes: Nous avons mené une étude de type CAP pendant 08 mois au Centre de Santé Marie Immaculée d'Etoug-Ebé à Yaoundé au Cameroun. L'échantillonnage était consécutif et exhaustif. Nous avons inclus dans notre étude des femmes enceintes âgées d'au moins 18 ans et étant à leur premier trimestre de grossesse. Au total 150 femmes enceintes ont participé à l'étude.

Résultats: $22,7 \%$ des femmes enceintes étaient obèses selon l'OMS. $10,7 \%$ avaient pris plus de poids que recommandé pendant la grossesse selon l'OIM. Parmi ces dernières, $43,8 \%$ avaient des attitudes mauvaises, $68,8 \%$ avaient une mauvaise pratique et $75 \%$ avaient des connaissances mauvaises sur l'obésité.

Conclusion: Il en ressort que les femmes enceintes de notre zone d'étude avaient des connaissances limitées au sujet de l'obésité ce qui expliquerait leurs mauvaises pratiques et attitudes.

Copy Right, IJAR, 2017,. All rights reserved.

\section{Introduction:-}

Ces dernières années, l'obésité dont les causes sont à la fois génétique, comportementale et environnementale a connu une évolution spectaculaire exigeant la mise en place des actions urgentes et concrètes des

Corresponding Author:- DAMARIS Enyegue Mandob.

Address:- Département des Sciences Biologiques, Ecole Normale Supérieure de l'Université de

Yaoundé I, Yaoundé, Cameroun, BP 047. 
responsables de la santé publique. Définie en 1997 par l'organisation mondiale de la santé (OMS) comme une accumulation anormale ou excessive de graisses corporelles pouvant nuire à la santé. L'obésité se mesure par différentes méthodes dont la plus utilisée en pratique clinique est la mesure de l'indice de masse corporelle (IMC) qui détermine la «corpulence» de l'individu. Un individu est considéré obèse dès lors que la valeur de l'IMC est supérieure à $30 \mathrm{Kg} / \mathrm{m}^{2}(1)$. Cette pathologie n'épargne aucune population et touche très directement les femmes enceintes d'autant plus que la période de grossesse est un moment au cours duquel les femmes sont généralement amenées à prendre du poids. Chez les gestantes, la prise excessive de poids est associée aux complications graves à court et à long terme, chez la mère et chez l'enfant, pendant la grossesse (hypertension artérielle, diabète gestationnel, déformation du tube neural du fotus); lors de l'accouchement (césarienne, dystocie de l'épaule) et après l'accouchement (dépression postpartum, maladies cardiovasculaires, obésité infantile) (2,3). La prévalence de l'obésité maternelle est variable d'un pays à un autre; elle était de 21,2\% au bangladesh (4), de 50\% en Australie et $36 \%$ aux Etats-Unis (5,6). En Afrique, cette prévalence s'observe beaucoup plus chez les mères âgées et multipares (7) ; Elle est de $10,7 \%$ au Nigéria (8) et de 44\% en Afrique du Sud (9). Au Cameroun, il y’a une absence des données relatives à ce sujet.

Malgré l'ampleur que prend cette maladie dans notre société surtout dans le secteur obstétrical, il n'existe pas de cadres d'éducation des femmes enceintes relatifs à l'obésité comme pour d'autres pathologies telles que le syndrome d'immunodéficience acquise (SIDA) et le paludisme (10). La gestion de la pandémie de l'obésité n'est donc possible que si les interventions efficaces de prévention sont menées au moment opportun. L'attention particulière de réduire l'obésité pendant la grossesse est essentielle non seulement pour le bien-être de la mère et celui du futur bébé mais aussi pour la santé de la nation et de son avenir. La diminution considérable des difficultés que posent l'obésité relève de l'implication personnelle de chaque gestante à prendre conscience de son état de santé et de celui de son enfant en essayant de maintenir son IMC à une valeur normale. Cette responsabilité individuelle ne peut s'exercer pleinement que là où les populations ont accès à des informations fiables, et sont soutenues pour faire les bons choix pour leur santé, ce qui n'est pas toujours le cas. Il est donc important pour les gestantes de connaitre les recommandations en matière de prise de poids ainsi que les complications en rapport avec le non-respect de celles-ci. Notre étude se propose d'évaluer la prévalence, les attitudes et connaissances relatives a l'obésité des femmes enceintes pendant les visites prénatales dans un centre de santé privé du Cameroun.

\section{Matériel Et Méthodes:-}

\section{Présentation du cadre de l'étude:-}

Notre étude dont l'objectif porte sur l'évaluation du niveau de connaissances des femmes enceintes sur l'obésité est de type Connaissances Attitudes et Pratiques. Cette étude a été menée au centre de santé Marie Immaculée situé au quatier Étoug-Ébé à Yaoundé de 7h30 à 14h30 les mercredi et le vendredi de la période allant d'Octobre 2016 à Mai 2017.

\section{Population d'étude}

Après cette causerie éducative relative à notre recherche, les gestantes étaient orientées vers la salle de consultation pour la prise des mesures anthropométriques (taille avec une toise, poids avec un pèse-personne de type SECA) selon les recommandations de L'OMS par une infirmière qui les mentionnaient dans leur carnet de santé. A la suite des examens physiques, les femmes devaient passer à l'interrogatoire.

Un questionnaire auto-administré comportant 4 sections est utilisé pour la collecte des données. La section 1 relative aux données sociodémographiques (nom, age, region d'origine, nombre de grossesses et d'enfants, profession, niveau d'étude, statut matrimonial)

La section 2 comprenait huit questions relatives aux connaissances des participantes sur l'obésité. La section 3 comprenait cinq questions relatives aux attitudes des participantes vis-à-vis de l'obésité. La section 4 comprenait 5 questions relatives à l'exploration des pratiques des participantes concernant l'obésité.

Le questionnaire élaborée par les enquêteurs été remis aux 150 patientes consentantes pour remplissage et remise le même jour. Les femmes qui n'étaient pas aptes à remplir le questionnaire se faisaient aider par le personnel compétent disponible ; au maximum quinze exemplaires étaient remplis par jour. 


\section{Analyses statistiques des données:-}

Les données ont été collectées grâce à une fiche technique conçue. Ensuite, elles ont été saisies dans un masque conçu dans le logiciel CsPro version 6.3. Ces données ont été analysées dans le logiciel SPSS version 23.0 pour Windows. Les graphiques ont été produits grâce au logiciel Microsoft Excel 2013. Les variables qualitatives ont été présentées avec les effectifs et les pourcentages. Les variables quantitatives ont été présentées avec le minimum, le maximum et la moyenne \pm Écart-type. Le niveau global de connaissances, d'attitudes et pratique a été évalué grâce à la somme des points obtenus pour chaque question de cette partie, un système de score a été proposé et le total a été réparti en tranches.

L'état des connaissances sur la notion de l'obésité chez la femme enceinte

0 point : connaissance nulle sur l'obésité ;

[1-4] point(s) : mauvaises connaissances sur l'obésité ;

5points : connaissances moyenne sur l'obésité ; [6-8] points: bonnes connaissances

pour les attitudes relatives à l'obésité

[0-2] point(s): mauvaises attitudes;

3 points: attitudes acceptables; [4-5] points : bonnes attitudes.

Pour l'exploration des pratiques relatives à l'obésité

[0-2] point(s): mauvaises pratiques ;

3 points : pratiques moyennes ; [4-5] points : bonne pratiques.

\section{Définition de l'obésité pendant la grossesse:-}

De ce fait, l'IMC des femmes enceintes est déterminé à partir du poids pré-grossesse. Ainsi les femmes dont l'IMC pré-gestationnel est supérieur à $30 \mathrm{~kg} / \mathrm{m}^{2}$ ont un risque d'avoir une grossesse associée à l'obésité (11). Ainsi, cet indice perd sa pertinence lorsqu'on le calcule pendant la grossesse. Par ailleurs, l'Institut De Médecine a établi en 1990 mais révisé en 2009 des recommandations relatives à la prise de poids pendant la grossesse selon leur IMC pré-gestationnel. Ainsi toute femme dont la prise de poids dépasse la valeur recommandée est identifiée comme développant l'obésité au cours de sa grossesse(OIM,2009).

\section{Résultats:-}

Les femmes ont été réparties en six classes d'âge. L'âge moyen de la population étudiée était de 27,37 $\pm 5,1$ ans, avec des extrêmes compris entre 19 ans et 48 ans. La tranche d'âge la plus représentée est celle de 25 à 30 ans avec un pourcentage de $37,3 \%$ et la moins représentée est celle dont l'âge est supérieur à 40 avec un pourcentage de $0,7 \%$ (Tableau 1).

22,7\% de la population étudiée a été identifiée comme obèse (tout type d'obésité confondu) ; 28,7\% comme étant en surpoids, 47,3\% comme étant normal et 1,3\% comme étant en maigreur (Tableau 2). La prise de poids recommandée selon l'OIM en fonction de l'IMC pré-gestationnel est respectée chez $89,3 \%$ de la population totale, beaucoup plus chez les gestantes présentant un IMC pré gestationnel normal. Par contre, parmi les 16(10.7\%) femmes dont la prise de poids a dépassé la valeur, recommandée la moitié d'entre elles avait déjà un IMC pré-gestationnel $\geq 30 \mathrm{~kg} / \mathrm{m}^{2}$ (Tableau 3). 43,8\% avaient des attitudes mauvaises, $68,8 \%$ avaient des mauvaises pratiques et $75 \%$ avaient des connaissances mauvaises sur l'obésité (Tableau 4).

Tableau 1:- Répartition des femmes enceintes en fonction de la tranche d'âge

\begin{tabular}{|l|l|l|}
\hline Tranche d'âge(ans) & Effectifs & Pourcentages (\%) \\
\hline$] 19-20]$ & 14 & 9,3 \\
\hline$] 20-25]$ & 40 & 26,7 \\
\hline$] 25-30]$ & 56 & 37,3 \\
\hline$] 30-35]$ & 32 & 21,3 \\
\hline$] 35-40]$ & 7 & 4,7 \\
\hline$>40$ & 1 & 0,7 \\
\hline
\end{tabular}

Tableau 2:- Répartition des femmes enceintes en fonction l'IMC pré-gestationnel

\begin{tabular}{|l|l|l|}
\hline IMC $\left(\mathbf{k g ~} / \mathbf{m}^{2}\right)$ & Effectifs & Pourcentages $\mathbf{( \% )}$ \\
\hline$<18$ & 2 & 1,3 \\
\hline
\end{tabular}




\begin{tabular}{|l|l|l|}
\hline$[18-25[$ & 71 & 47,3 \\
\hline$[25-30[$ & 43 & 28,7 \\
\hline$\geq 30$ & 34 & 22,7 \\
\hline
\end{tabular}

Tableau 3:- Répartition des femmes enceintes en fonction du respect ou non des normes OIM de prise de poids au cours de la grossesse selon les tranches d'IMC pré-gestationnel

\begin{tabular}{|l|l|l|}
\hline IMC $\left(\mathbf{k g} / \mathbf{m}^{\mathbf{2}}\right)$ & $\begin{array}{l}\text { Effectifs et Pourcentages }(\%) \text { des } \\
\text { femmes respectant la norme de } \\
\text { prise de poids OIM }\end{array}$ & $\begin{array}{l}\text { Effectifs et Pourcentages }(\%) \text { des } \\
\text { femmes ne respectant la norme de } \\
\text { prise de poids OIM }\end{array}$ \\
\hline$[18-25[$ & $68(95,8)$ & $3(4,2)$ \\
\hline$[25-30[$ & $38(88,4)$ & $5(11,6)$ \\
\hline$\geq 30$ & $26(76,5)$ & $8(23,5)$ \\
\hline Total & $134(89,3)$ & $16(10,7)$ \\
\hline
\end{tabular}

Tableau 4:- Connaissances, Attitudes et Pratiques des femmes enceintes vis-à-vis de l'obésité

\begin{tabular}{|c|c|c|}
\hline Variables & Effectifs & Pourcentages (\%) \\
\hline \multicolumn{3}{|l|}{ Attitudes } \\
\hline Mauvais & 7 & 43,8 \\
\hline Acceptable & 5 & 31,2 \\
\hline Bon & 4 & 25 \\
\hline \multicolumn{3}{|l|}{ Pratiques } \\
\hline Mauvaise & 11 & 68,7 \\
\hline Moyenne & 4 & 25 \\
\hline Bonne & 1 & 6,2 \\
\hline \multicolumn{3}{|c|}{ Connaissances } \\
\hline Nulle & 1 & 6,2 \\
\hline Mauvaise & 12 & 75 \\
\hline Moyenne & 2 & 12,5 \\
\hline Bonne & 1 & 6,2 \\
\hline
\end{tabular}

\section{Discussion:-}

L'utilisation des services de santé est liée à de nombreuses autres influences ayant trait aux populations comme a pauvreté, la réceptivité à l'endroit de la médecine moderne, l'existence des assurances médicales (13).

L'âge moyen de notre population d'étude était 27,37 $\pm 5,1$ ans. Par ailleurs, une autre étude menée par Njoya et ses collaborateurs en 2013 à l'hôpital de la Cité Verte dont l'un des objectifs était de déterminer aussi les caractéristiques sociodémographiques des femmes enceintes en visite prénatale a montré que l'âge moyen de la population étudiée était de 24,54 \pm 6 ans (14). En comparant nos résultats à ceux de Njoya et ses collaborateurs, nous constatons une légère différence sur l'âge moyen des femmes enceintes à Yaoundé, cela pourrait être due au fait que dans la seconde étude, la population était plus grande (273 patientes) et un peu plus jeune car il a été inclus des gestantes âgées de 16 ans ce qui n'est pas le cas dans notre étude.

$22,7 \%$ des femmes ayant participé à l'étude ont été identifiées comme obèses. Ces résultats sont aussi élevés que ceux rapportés par les études antérieures( 4, 15) ( 21,2\%) dans leurs travaux respectivement au Bangladesh et en Australie , mais cependant bas par rapport que ceux rapportés par Abdel et Adel en 2009 (28,6\%), Basu et ses collaborateurs en 2010 (44\%) respectivement en Arabie saoudite et en Afrique du Sud $(9,16)$.

En effet, parmi les 353 gestantes ayant participé à l'étude de Shub, seules 21,2\% ont été classées obèses. Ceci montre que l'obésité qui est présent dans les pays développés se repend de plus en plus dans les pays en voie de développement et que la plupart des femmes en âge de procréer sont en surpoids ou obèse (17, 18). Les études menées sur les populations urbaines du Cameroun ont montré que l'obésité est de plus en plus répandue chez les adultes et évolue au fil du temps. La prévalence de l'obésité était estimée en 2002 à 17,1\% chez les femmes urbaines Camerounaises (19) et augmente chez les femmes en âge de procréer (20). Par ailleurs, la plupart des grossesses sont 
non planifiées au Cameroun et les femmes en surpoids et obèses n'ont pas souvent la possibilité de normaliser leur IMC avant et pendant la grossesse (21) d'où cette différence de résultats.

Cette prévalence est aussi élevée en Afrique du Sud. C'est l'un des pays les plus développés en Afrique dont l'urbanisation rapide et le développement socio-économique entraîneraient une occidentalisation du mode de vie. En effet, les régimes alimentaires traditionnels (souvent peu diversifiés et limités en quantité) en Afrique qui reposent sur les céréales, les racines et tubercules, peu de produits d'origine animale, les aliments avec une faible proportion de lipides sont progressivement remplacés par des régimes plus abondants et variés lorsque les revenus moyens s'élèvent. L'urbanisation, la mise à disposition de produits moins fastidieux à préparer, l'accès plus facile à des produits d'origine animale contribuent alors à une modification sensible de l'alimentation. On parle d'une occidentalisation de l'alimentation (22).

Dans les deux cas, Il a été prouvé par l'OMS que la première cause de l'obésité reste un déséquilibre alimentaire associé à une inactivité physique. En Afrique en général et au Cameroun en particulier les populations souffrirait de la malnutrition et dans les pays occidentaux, ce régime serait riche en aliments transformés et importés ce qui réduit l'utilisation des légumes et fruits frais produits localement. En plus, seulement 10,7\% des femmes ont dépassé la prise de poids recommandée par l'Institut De Médecine (12). Ce taux est moins élevé que celui de 35,5\% retrouvé dans une étude menée à l'hôpital gynéco-obstétrique et pédiatrique de Yaoundé par Ahounkeng et ses collaborateurs en 2014 car notre échantillon était principalement constitué des femmes à leur premier trimestre de grossesse tandis que leur population d'étude était constituée de femmes soit en travail, soit en post-partum ou en post-césarienne c'est- à-dire des femmes à la fin du troisième trimestre de grossesse ou ayant déjà accouché (23). Or si l'on définit le gain pondéral durant la grossesse comme étant la différence entre le poids en début de grossesse et le poids en fin de grossesse, il serait évident que la prise de poids chez les femmes enceintes n'est pas effective au premier trimestre de grossesse.

Dans l'échantillon étudié, la majorité des femmes ayant un gain pondéral excessif durant la grossesse avaient une connaissance limitée de l'obésité surtout en ce qui concerne le calcul de l'IMC, le nombre de kilogramme à gagner et les problèmes liés à l'obésité pendant la grossesse. Ces résultats sont proches de ceux trouvés par : Gaudet ses collaborateurs en 2011 lors d'une étude réalisée au Canada indiquant que la plupart des femmes étaient incapable d'identifier les complications liées à l'obésité aussi bien pour la mère que pour le bébé (24); Thompson et ses collaborateurs qui ont plutôt trouvé dans une étude réalisée en 2011 en Nouvelle Zélande que 70,5\% des femmes étaient incapables d'identifier la quantité de poids à prendre pendant la grossesse (25). Shub et ses collaborateurs en 2013 en Australie attestant que les connaissances des femmes sur l'obésité en général, la détermination de l'IMC et des risques spécifiques liés à l'excès de poids pendant la grossesse en particulier étaient pauvres (15).

Le point commun de toutes ces études est que la plupart des femmes enceintes avaient tendance à sous-estimer leur IMC. Or, la perception des menaces de santé reliée au poids est décroissante chez les individus qui sous-estiment leur IMC. Ceci pouvant conduire à la réduction de la recherche des connaissances et de la pratique des attitudes saines qui diminuent les risques d'être atteints par cette maladie (26); par conséquent entrainer une perception inexacte de l'impact du poids sur les futures grossesses $(27,28$,$) .$

Dans notre étude aucune femme n'a cité la déformation du tube neural comme conséquence de l'obésité maternelle sur le fœetus; et dans beaucoup d'autres études cette anomalie n'est pas citée comme risque associé à l'obésité car le risque d'avoir les anomalies du tube neural chez le foetus de mère obèse peut être réduit en prenant une dose surnuméraire de $5 \mathrm{mg}$ d'acide folique par jour avant la conception et pendant le premier trimestre de la grossesse (29).

Concernant les attitudes des femmes enceintes vis-à-vis de l'obésité, nous dirons qu'elles sont dérisoires car l'on a observé que la moitié de notre population d'étude avait un comportement alimentaire déplaisant et plus de la moitié avait une mauvaise approche diététique. Nos résultats se rapprochent de ceux obtenus à la suite d'une enquête menée par Prakash Simkhada et ses collaborateurs en 2011 au Népal montrant que plus de la moitié de la population adoptait un régime alimentaire à risque bien qu'ayant des connaissances sur l'obésité (30).

Malgré l'obtention des résultats presque similaires, notons qu'il n'existe pas encore au Cameroun en général et à Yaoundé en particulier des programmes effectifs de prévention de l'obésité maternelle qui pourrait inciter les 
femmes enceintes à éviter des comportements à risque vis- à -vis de cette maladie. En revanche au Népal, malgré les connaissances solides que possède la population sur l'obésité, il en ressort de cette étude que les népalaises considèrent l'obésité comme un signe de prospérité et qu'elles sont très paresseuses, d'où leur inactivité physique.

\section{Conclusion:-}

Il en ressort que l'obésité maternelle existe en proportion appréciable dans notre zone d'étude (22,7\%) avec une minorité des femmes enceintes $(10,7 \%$ ) ayant un gain pondéral excessif au cours de leur premier trimestre de grossesse. La majorité de ces gestantes avaient des mauvaises connaissances, mauvaises attitudes et mauvaises pratiques vis-à-vis de l'obésité.

\section{Remerciements:-}

Les auteurs remercient les participantes à cette 2tude et tout le personnel du centre de santé Marie-Immaculée d'Etoug-Ebé.

\section{Conflit d'ntérêt:-}

The authors declare that there are no conflicts of interest.

\section{Funding:-}

This research received no specific grant from any funding agency in the public, commercial, or not for profit sectors.

\section{Reférences:-}

1. Organisation Mondiale de la Santé.(1997) preventing and managing the global epidemic.Report of WHO Consultation on Obesity.

2. Rode L., Hegaard H.K., Kjaergaard H., Møller L.F., Tabor A., Ottesen B. (2007) Association between maternal weight gain and birth weight.Obstetrics Gynecology.109: 1309-15.

3. Crane J.M.G., White J., Murphy P., Burrage L., Hutchens D. (2009) The effect of gestational weight gain by body mass index on maternal and neonatal outcomes. Journal Obstetrics Gynaecology. 31: 2835.Diez Roux A.V., Mair C. (2010) Neighborhoods and health. Ann N Y Acad Sci. 1186: 125-45.

4. Statabdi Goon. (2013) Prevalence of obesity among Bangladeshi pregnant women at their first trimester of pregnancy.Central Asian Journal of Global health. 2 (2)

5. Dodd J.M., Grivell R.M., Nguyen A.M., Chan A., Robinson J.S. (2011) Maternel and perinal health outcomes by body mass index category. Aust NZJ obstet gynaecol 51: 136-140.

6. Flegal K.M., Carroll M.D., Kit B.k., Odgen, C.L. (2012) prevalence of obesity body mass index among US adults, 1999-2010. JAMA 307: 491-497.

7. Ojochenemi J., Onubi Debbi Marais, Lorna Aucott, Friday Okonofua, Amudha Poobalan . (2016) Maternal obesity in Africa: a systematic review and meta-analysis. J Public Health (Oxf) 38 (3) : 218-231.

8. Chigbu C.O., Aja L.O. (2011) Obesity in pregnancy in southeast Nigeria. Annals of Medecine and Health Sciences Research 1 (2) : 135-40.

9. Basu J.K., Jeketera C.M., Basu D. (2010) Obesity and its outcomes among pregnant South African women. Int J Gynecol Obstet. 110, (2) :101-4.DOI:10.1016/j.ijgo.2010.02.020. Epub 2010 Apr 24.

10. Organisation Mondiale de la Santé (OMS) Génève : 2004.Recommandations pour les enquêtes sérologiques sentinelles concernant le VIH : femmes enceintes et autres groupes.

11. Catalano P.M., Mele L., Landon M.B. (2014) Inadequate weight gain in overweight and obese pregnant women: what is the effect on fetal growth? American Journal of Obstetrics and Gynecology. pii: S0002-9378 (14)00121-5. doi:10.1016/j.ajog.2014.02.004.

12. Institute of Medicine. Weight gain during pregnancy: reexamining the guidelines. Washington, D.C: The National Academies Press; 2009

13. Fournier P., Haddad D. (1995) Les facteurs associés à l'utilisation des services de santé dans les pays en developpement.In Hubert G. , Piche V. La sociologie des populations Montréal, AUPELF-UREF 289325.

14. Njoya O. Essi M.J., Ongolo B., Obama M.T. (2013) Connaissances, Perceptions et Pratiques des Femmes Enceintes Vis-à-vis de l'Hépatite Virale B en Milieu Urbain au Cameroun. Health Sciences 14 (2) : 12. 
15. Shub A, Emily Y-S Huning, Karen J Campbell, Elizabeth A. (2013) McCarthy. Pregnant women's knowledge of weight, weight gain, complications of obesity and weight management strategies in pregnancy. BMC Research Notes 6:278.

16. Abdel-Hady El-Gilany, Adel El-Wehady. (2009) Prevalence of Obesity in a Saudi Obstetric Population.Obesity Facts (2): 217-220. DOI: 10.1159/000226597.

17. Linné, Y. (2004) Effects of obesity on women's reproduction and complications during pregnancy. Obesity Review. (5) :137-143.

18. Young V.R. (2001) Good nutrition for all: challenge for the nutritional sciences in the new millennium. Nutrition Today, (36) :6-16.

19. Sobngwi E., Mbanya J., Unwin N., Kengne A., Fezeu L., Minkoulou E. (2002) Physical activity and its relationship with obesity, hypertension and diabetes in urban and rural Cameroon. International Journal of Obesity 26: 1009-1016.

20. Yee L.M., Cheng Y.W., Inturrisi, M., Caughey A.B. (2011). Effect of gestational weight gain on perinatal outcomes in women with type 2 diabetes mellitus using the 2009 Institute of Medicine guidelines. American Journal of Obstetrics \& Gynecology 205:257-261.

21. Kamadjeu R., Edwards R., Atanga J., Kiawi E., Unwin N., Mbanya J. (2006) Anthropometry measures and prevalence of obesity in the urban adult population of Cameroon: An update from the Cameroon Burden of Diabetes Baseline Survey. BMC Public Health $6: 228$.

22. Popkin B.M. (2001)The nutrition transition and obesity in the developing world.The journal of nutrition 131 (3) : 8715-8735.

23. Ahounkeng N.P., Mboudou E.T., Adjoby C.R., Rakotomalala N.Z., Foumane P., Dohbit S.J., Nshimirimana E.T. (2014) Impact du gain pondéral excessif pendant la grossesse sur l'issue maternofœtale à l'hopital gynéco-obstétrique et pédiatrique de Yaoundé (Cameroun). Med Sante Trop. (24): 6367. DOI : $10.1684 / \mathrm{mst} .2014 .0290$.

24. Gaudet L., Gruslin A. and Magee L. (2011) Weight in pregnancy and its implications: what women report? Journal of Obstetrics and Gynaecology Canada 33 (3) : 227-234.

25. Thompson M. (2011) Pregnant women's knowledge of obesity and ideal weight gain in pregnancy, and health behaviours of pregnant women and their partners. The Australian and New Zealand Journal of Obstetrics and Gynaecology 51 (5): 460-463.

26. Post R. (2011) The influence of physician acknowledgment of patients' weight status on patient perceptions of overweight and obesity in the United States. Archives of Internal Medicine 171 (4) : 31632.

27. Krishnamoorthy U., Schram C., Hill S. (2006) Maternal obesity in pregnancy: Is it time for meaningful research to inform preventive and management strategies? British Journal of Obstetrics and Gynaecology 113 (10) :1134-1140.

28. Yu C., Teoh T.,Robinson S. (2006) Obesity in pregnancy. British Journal of Obstetrics and Gynaecology 1117-1125

29. Modder J. (2010) CMACE/RCOG Joint Guideline. Management of Women with Obesity in,Pregnancy. 